\section{Comparison of CBD and Cannabis Use IMotives}

\section{Aksinya Bilaonova ${ }^{1}$, Joy A. Phillips ${ }^{2}$, Kristen G. Anderson, Ph.D. ${ }^{1}$}

${ }^{1}$ Adolescent Health Research Program, Department of Psychology, Reed College, Portland, Oregon

2 The Donald P. Shiley BioScience Center'San Diego State University, San Diego, California
Cannabis

2021, Volume 4 (2)

(C) Author(s) 2021

researchmj.org

DOI: 10.26828/cannabis/2021.02.005

\begin{abstract}
Cannabidiol, also known as CBD, has increasingly gained popularity as a cure-all product and is now found in products across a variety of industries. Despite the surge in popularity, little remains known about individual motives and patterns of CBD use. The goal of this study was to gain a better understanding of the similarities and differences between motives for CBD and cannabis use as well as comparing motives for younger and older users. Participants $(N=171)$ in U.S. states and territories with legalized cannabis for recreational use were recruited via Amazon MTurk and were asked to complete an anonymous survey assessing their CBD and cannabis use, effects, and motives for use. The greatest differences between self-reported CBD and cannabis use were for side effect profiles. While the recreational use motive was more commonly endorsed for cannabis, use for beauty purposes was more common for CBD. No age group differences emerged for motives to use CBD or cannabis. Future research examining age-related differences in a larger, more age-diverse samples is recommended.
\end{abstract}

Key words: cannabidiol; CBD; cannabis; motives

Cannabis sativa L (Cannabis spp. or Cannabis) is one of the world's oldest cultivated plants, commonly known as marijuana or hemp (Russo, 2007). Over 120 cannabinoids have been identified in cannabis (Miller \& Miller, 2017). The most abundant cannabinoid, tetrahydrocannabinol (THC), is known for its psychoactive properties due to its ability to act as a partial agonist for CB1 cannabinoid receptors (National Academies of Sciences, 2017). Cannabidiol, also known as CBD, is the second most abundant cannabinoid and does not appear to trigger the high or euphoria associated with THC (Shannon et al., 2019). CBD is currently licensed for treatment of two pediatric seizure disorders (Greenwich Biosciences, Inc., 2018). Due to its low toxicity and potential antioxidant and anti-inflammatory properties, CBD is currently the subject of multiple clinical trials (Larsen \& Shahinas, 2020).

The regulatory status of CBD in the U.S. remains complicated. One of the most important factors in determining the legality of CBD is its source (Corroon \& Kight, 2018). While cannabis plants contain high concentrations of THC and are grown for recreational use, hemp plants are industrially grown for their fibers and contain less than $0.3 \%$ THC, making them federally legal (Shannon et al., 2019). The regulatory status of CBD thus depends on its source, with cannabisderived CBD considered a Schedule I controlled substance by the Controlled Substances Act (U.S. Drug Enforcement Administration, n.d.). However, hemp-derived CBD is legal under federal law and the laws of some states as long as it contains less than $0.3 \%$ THC (Corroon \& Kight, 2018). The situation is further complicated by the absence of strict FDA enforcement, often leading to the mislabeling of CBD products, with a number of CBD products available on the market being under- and over-labeled (Bonn-Miller et al., 2017). Some CBD products also contain trace amounts of THC, sufficiently high to produce intoxication or impairment (Bonn-Miller et al., 2017).

The popularity of $\mathrm{CBD}$ is on the rise. The number of CBD-related Google searches has increased exponentially each year starting from 2014 (Leas et al., 2019). Sale reports point to the rising interest in CBD products, with projected retail sales expected to reach up to $\$ 20$ billion by 
2024 (Boulder, 2019; Corroon \& Kight, 2018). CBD products exist in a variety of industries, including cosmetic and skincare products, food and beverages, health and wellness products and supplements, pet products, perfume, and even clothing and bedding (Boulder, 2019). Currently, hemp-derived CBD products can be purchased both online and over the counter throughout most of the U.S. Cannabis-derived CBD products, however, can only be purchased in specialized cannabis dispensaries by qualifying patients in the states where medical cannabis is legal and by customers in states where recreational cannabis is legal (Corroon \& Kight, 2018).

CBD has been marketed online as a cure-all for a variety of health problems, including serious long-term conditions such as cancer, diabetes, and HIV/AIDS. Understanding motives and patterns of CBD use can thus help shed light on why individuals might use CBD despite its quasiillegal status and the current scarcity of scientifically demonstrated health benefits. Corroon and Phillips (2018), one of the first studies on CBD motives, found that over $60 \%$ of participants were using CBD to treat a medical condition; in the order of frequency, the top medical conditions reported were pain, anxiety and depression, and insomnia. Similarly, Fedorova et al. (2021) concluded that CBD use among young adults was associated with motivations linked to pain and medical and psychological conditions. Zenone et al. (2021) found that CBD consumers were primarily motivated by internal reasons, such as an unmet medical need that was not satisfied by other treatment options.

Although there is a significant overlap between recreational and medical cannabis users, medical cannabis users tend to use cannabis primarily to treat a variety of medical and psychiatric problems. Vedelago et al. (2020) found that recreational cannabis use was primarily driven by positive reinforcing aspects of cannabis, such as enjoyment and celebration, while those who used cannabis for medicinal purposes predominantly reported using for negative reinforcing aspects such as social anxiety and sleep. Medicinal cannabis is most frequently used to treat pain, anxiety, stress, depression, headache/migraine, nausea, insomnia, and muscle spasticity, with some patients seeking cannabis enriched with CBD (Bonn-Miller et al.,
2014; Sexton et al., 2016). Individuals are seemingly using cannabis for a wide range of medical conditions, including the ones for which there is no formal research to support the use (e.g. depression and anxiety). While the main motives to use medical cannabis seem to be relatively consistent across different studies, individuals may differ both in their motives for use and the outcomes of use based on age and when regular use begins (Haug et al., 2017).

\section{Current Research}

Although CBD products seem to be commonly used, the data on individual motives and patterns of CBD use remains scarce. Previous literature on CBD use motives focused primarily on examining health-related motives for CBD use and did not account for the relatively recent surge in popularity of cosmetic and wellness products containing CBD. No previous studies have compared $\mathrm{CBD}$ and cannabis (i.e., cannabis containing THC) motive endorsement for the same set of reasons to use. Finally, little is known about the potential differences in CBD use motives among older and younger populations.

One goal of this study was to better understand the similarities and differences between motives for CBD and cannabis use. Based on the findings by Corroon and Phillips (2018), we expected the most frequently reported reasons for CBD use to include management of medical and psychiatric symptoms, such as pain management and joint treatment, depression, anxiety, and problems with sleep. We also extended findings from Haug et al. (2017) by examining possible agerelated differences in motives to use $\mathrm{CBD}$ and cannabis. Older participants may be more likely than younger participants to report using CBD for medical conditions, such as chronic pain and joint treatment, since those conditions tend to arise at an older age. With an estimated mean age of onset of 21.3 years for anxiety disorders (Lijster et al., 2017), and 25-45 for the onset of mood disorders (Kessler et al., 2007), younger participants might be more likely than older participants to use CBD for psychiatric reasons, such as management of anxiety and depression. The recent rise in popularity of CBD in cosmetic and wellness products may highlight new motives not previously assessed. Finally, we also examined characteristics of CBD and cannabis use, such as 
reported side effects, preferred formats of CBD and/or cannabis products, and the cost of use.

\section{METHODS}

\section{Participants}

Using Amazon's MTurk, 190 participants residing in cannabis legal states within in the US (Alaska, California, Colorado, District of Columbia, Maine, Massachusetts, Michigan, Nevada, Oregon, Vermont, and Washington), over 21 years of age, and had used cannabis and/or CBD in the past 30 days were recruited. Participants who provided inappropriate or nonsensical answers to the open-ended questions were dropped from the dataset to reduce the influence of random responding $(n=19)$. Nonsensical responses were defined as any responses that were inappropriate, unrelated, incomprehensible, or incoherent. The final sample $(N=171)$, including both CBD $(n=109)$ and cannabis users $(n=161)$ was predominantly white, identified as men, between the ages of $21^{-}$ 54 , residing in California. Over half of the sample endorsed using both CBD and cannabis products (Table 1). Socioeconomically, participants most commonly had some college education/degree and fit within the middle class income level. Modal use frequency for CBD was 1-2 times per week (23.5\%; $n=27)$ and 6-7 times per week $(23.5 \% ; n=27)$ with the majority of participants using weekly or more $(69.6 \% ; n=80)$. For cannabis, the most common use frequency was 1-2 days per week $(21.6 \% ; n=30)$; while a sizable proportion used multiple times per day $(16.6 \% ; n=23), 69 \%(n=$ 96) used weekly or more.

\section{Measures}

Demographic questions assessed participants' age, gender, race and ethnicity, education level, household income, and the U.S. state of residence.

Use patterns and reasons to use CBD and medical cannabis were assessed using the 104item CBD and Cannabis Use Motives Checklist, adapted from a questionnaire created by Corroon and Phillips (2018) to include questions about both CBD and cannabis use. Side effects of CBD (range: $0-13 ; \Omega=0.81$ ) and cannabis use (range: $0-11 ; \Omega=0.79$ ) listed 14 potential effects for each, such as dry mouth, dizziness, and balance issues
(Table 2). Euphoria, a side effect of CBD but a desired effect for cannabis, was included in the measurement of CBD side effects but not cannabis. Confirmatory factor analysis (CFA) of the side effects scales, using item-total parcels as indicators due to sample size constraints (4 parcels each), demonstrated excellent fit to the data; CBD: $x^{2}(d f=2)=1.98, p=.37$; RMSEA $=$ .00 (CI: $.00-.19), p$ close $=.46 ; \mathrm{CFI}=1.00 ; \mathrm{SRMR}$ $=.03, n=109$; cannabis: $x^{2}(d f=2)=0.24, p=$ $.89 ; \mathrm{RMSEA}=.00$ (CI: .00 - .07), $p$-close $=.92 ; \mathrm{CFI}$ $=1.00 ; \mathrm{SRMR}=.006, n=161$. A single-item assessed the cost of CBD and/or cannabis use (\$/week), and a multiple choice item queried preferred format of CBD and/or cannabis products (i.e., What format of $C B D$ and/or cannabis do you use?; e.g., smoking, edibles).

Checklist items $(0=$ absent $; 1=$ present $)$ identified four general reasons for using (medical condition, general health and well-being, recreational use, beauty/skincare) for CBD and cannabis separately. Individual reasons for use included health conditions for which participants use CBD and/or cannabis (20 medical; 8 psychiatric; Table 3). Health reasons were summed for CBD and cannabis separately (CBD: range $=0-15 ; \Omega=0.89 ;$ cannabis: range $=0-17 ; \Omega$ $=0.89$ ). CFA (3 medical parcels; 2 psychiatric) supported a single latent variable for CBD health motives, $x^{2}(d f=5)=6.62, p=.25$; RMSEA $=.06$ (CI: .00 - .15), $p$-close $=.39 ; \mathrm{CFI}=0.99 ; \mathrm{SRMR}=$ $.04, n=109$, and for cannabis health motives, $x^{2}(d f=5)=6.27, p=.28$; RMSEA $=.04(\mathrm{CI}: .00$ $.12), p$-close $=.49 ; \mathrm{CFI}=1.00 ; \mathrm{SRMR}=.03, n=$ 161 , both models demonstrating good to excellent fit.

The frequency of use and daily cannabis use among cannabis users was assessed using the Daily Sessions, Frequency, Age of Onset, and Quantity of Cannabis Use Inventory (DFAQ-CU; Cuttler \& Spradlin, 2017). DFAQ-CU is a 24-item, 6 -factor questionnaire. We validated motives scores against the daily use $(\alpha=0.70)$ and frequency scales $(\alpha=0.83)$.

\section{Procedure}

The college institutional review board approved all study procedures. MTurk respondents were redirected to the survey on a secure Qualtrics website. After asserting consent, participants provided demographic information 
and responded to the $\mathrm{CBD}$ and Cannabis Use Motives Checklist and DFAQ-CU in randomized order. After debriefing, which included substance use resources, participants were paid $\$ 3$ for 15 minutes of participation.

Table 1. Sample Demographic Characteristics $(N=171)$

\begin{tabular}{|c|c|c|c|}
\hline & $\%(n)$ & & $\%(n)$ \\
\hline Gender & & State of Residence & \\
\hline Men & $55.4(93)$ & $\mathrm{CA}$ & $45.6(78)$ \\
\hline Women & $44.0(74)$ & $\mathrm{CO}$ & $7.0(12)$ \\
\hline Non-binary & $0.6(1)$ & $\mathrm{DC}$ & $0.6(1)$ \\
\hline Age & & $\mathrm{ME}$ & $4.1(7)$ \\
\hline $21-24$ & $41.2(71)$ & MA & $8.8(15)$ \\
\hline $25-54$ & $46.2(79)$ & MI & $18.7(32)$ \\
\hline $55+$ & $12.3(21)$ & OR & $4.1(7)$ \\
\hline Race & & VT & $1.8(3)$ \\
\hline White & $81.8(139)$ & WA & $4.7(8)$ \\
\hline Black/African-American & $5.3(9)$ & Income & \\
\hline American Indian/Native American & $0.6(1)$ & $<\$ 35,000$ & $32.4(55)$ \\
\hline Asian/Pacific Islander & $11.2(19)$ & $\$ 35 \mathrm{~K}-74,999$ & $43.5(74)$ \\
\hline Other & $1.2(2)$ & Over $\$ 75,000$ & $24.1(41)$ \\
\hline Latinx & $9.4(16)$ & Medical Card & $20.0(31)$ \\
\hline Education & & Use Group & \\
\hline Some HS & $1.3(2)$ & CBD-Only & $5.9(10)$ \\
\hline HS Graduate & $15.2(26)$ & Can-Only & $36.2(62)$ \\
\hline Tech/Voc. Ed. & $2.9(5)$ & $\mathrm{CBD}+\mathrm{Can}$ & $57.9(99)$ \\
\hline Some College & $33.3(57)$ & & \\
\hline College Graduate & $47.4(81)$ & & \\
\hline
\end{tabular}

Note: $H S$ = high school; Tech/Voc Ed = trade/technical/vocational training; Medical Card = cannabis medical card holder; $C B D$-only = uses cannabidiol (CBD) only; Can-Only = use cannabis only; $\mathrm{CBD}+\mathrm{Can}=$ uses CBD and cannabis; State of Residence $=$ the cannabis legal ${ }^{-}$ state where the participant resides (US postal abbreviations); totals may not sum to $100 \%$ due to rounding or incomplete data. 
Table 2. Self-Reported Use Format, Side Effects, and Cost for CBD vs. Cannabis $(N=171)$

\begin{tabular}{|c|c|c|c|c|}
\hline & $\frac{\underline{C B D}}{\underline{(n=109)}}$ & $\frac{\text { Cannabis }}{\frac{(n=161)}{\underline{\%(n)}}}$ & $\underline{z / t}$ & $p$ \\
\hline Use Format & $M=5.5 ; S D=2.4$ & $M=3.1 ; S D=2.2$ & 8.2 & $<0.0001$ \\
\hline Smoking & $14.7(16)$ & $70.8(114)$ & -9.1 & $<0.0001$ \\
\hline Vaping & $26.6(29)$ & $37.9(61)$ & -1.9 & 0.05 \\
\hline Edibles & $37.6(41)$ & $46.0(74)$ & -1.4 & 0.17 \\
\hline Pill & 17.4(19) & $5.6(9)$ & 3.1 & 0.002 \\
\hline Sublingual & $34.9(38)$ & $6.2(10)$ & 6.0 & $<0.0001$ \\
\hline Topical & $34.9(38)$ & $1.9(3)$ & 7.4 & $<0.0001$ \\
\hline Liquid & 21.1(23) & $3.7(6)$ & 4.5 & $<0.0001$ \\
\hline Clothes & $7.3(8)$ & $5.0(8)$ & 0.8 & 0.42 \\
\hline Cosmetics & 11.9(13) & $1.9(3)$ & 2.6 & 0.01 \\
\hline Side Effects & $M=1.7 ; S D=0.2$ & $M=3.1 ; S D=0.2$ & -4.6 & $<0.0001$ \\
\hline Red eyes & $11.0(12)$ & $51.6(83)$ & -6.8 & $<0.0001$ \\
\hline Anxiety & $8.3(9)$ & $13.0(21)$ & -1.2 & 0.22 \\
\hline Calmness/relax & $58.7(64)$ & $52.8(85)$ & 1.0 & 0.34 \\
\hline Sensitivity & $11.0(12)$ & $17.4(28)$ & -1.4 & 0.15 \\
\hline Dry mouth & $10.1(11)$ & $43.5(70)$ & -5.9 & $<0.0001$ \\
\hline Stomach problems & $2.8(3)$ & $3.1(5)$ & -0.2 & 0.87 \\
\hline Sleepiness & $26.6(29)$ & $41.6(67)$ & -2.5 & 0.01 \\
\hline Poor concentration & $6.4(7)$ & $23.0(37)$ & -3.6 & 0.0003 \\
\hline Hunger/overeating & $3.7(4)$ & $40.4(65)$ & -6.8 & $<0.0001$ \\
\hline Increased heart rate & $1.8(2)$ & 11.2(18) & -2.9 & 0.004 \\
\hline Headache & $3.7(4)$ & $3.7(6)$ & -0.0 & 0.98 \\
\hline Dizziness & $7.3(8)$ & $6.2(10)$ & 0.4 & 0.71 \\
\hline Impaired balance & $2.8(3)$ & $7.5(12)$ & -1.7 & 0.10 \\
\hline $\begin{array}{l}\text { Perceptual } \\
\text { disturbance }\end{array}$ & $3.7(4)$ & $13.0(21)$ & -2.6 & 0.009 \\
\hline Euphoria/high* & $11.9(13)$ & $64.6(104)$ & -8.6 & $<0.0001$ \\
\hline Cost per week (\$) & $M=25.8 ; S D=30.6$ & $M=35.8 ; S D=46.2$ & -1.9 & 0.06 \\
\hline
\end{tabular}

Note: Edibles = consumption in food or beverages; $*$ Euphoria was listed as a side effect of CBD use; however, it was not included in the total number of side effects for either CBD or cannabis in this analysis as it may be the desired effect of cannabis use; Statistically significant differences highlighted in bold (Bonferroni correct $p<.0019$ ). $z$ derived from proportions test. 
Table 3. Reasons for Using $C B D$ and Cannabis $(N=171)$

\begin{tabular}{|c|c|c|c|c|}
\hline & $\frac{\frac{C B D}{(n=109)}}{\underline{\%(n)}}$ & $\frac{\frac{\text { Cannabis }}{(n=161)}}{\underline{\%(n)}}$ & $\underline{z / t}$ & $\underline{p}$ \\
\hline Beauty & $21.1(23)$ & $2.5(4)$ & 5.0 & $<0.001$ \\
\hline General health & $63.3(69)$ & $44.1(71)$ & 3.10 & 0.002 \\
\hline Health Cond. & $M=0.6 ; S D=1.7$ & $M=0.5 ; S D=1.6$ & -0.5 & 0.65 \\
\hline AIDS & $1.8(2)$ & $0.6(1)$ & 0.9 & 0.35 \\
\hline Arthritis & $11.0(12)$ & $7.5(12)$ & 1.0 & 0.31 \\
\hline Brain Injury & $2.8(3)$ & $3.1(5)$ & -0.2 & 0.87 \\
\hline Cancer & $1.8(2)$ & $1.9(3)$ & -0.0 & 0.99 \\
\hline Chronic fatigue & $4.6(5)$ & $1.9(3)$ & 1.3 & 0.20 \\
\hline Chronic pain & $20.2(22)$ & $11.8(19)$ & 1.9 & 0.06 \\
\hline Diabetes & $4.6(5)$ & $3.7(6)$ & 0.4 & 0.73 \\
\hline Epilepsy & $3.7(4)$ & $1.9(3)$ & 0.9 & 0.36 \\
\hline Fibromyalgia & $8.3(9)$ & $3.1(5)$ & 1.9 & 0.06 \\
\hline GI & $2.8(3)$ & $2.5(4)$ & 0.1 & 0.90 \\
\hline Glaucoma & $2.8(3)$ & $1.2(2)$ & 0.9 & 0.37 \\
\hline Hormonal & $8.3(9)$ & $3.7(6)$ & 1.6 & 0.11 \\
\hline Migraine & $7.3(8)$ & $8.1(13)$ & -0.2 & 0.82 \\
\hline Nausea & $5.5(6)$ & $3.7(6)$ & 0.7 & 0.49 \\
\hline Neuralgia & $2.8(2)$ & $1.9(3)$ & 0.5 & 0.63 \\
\hline Parkinson's & $1.8(2)$ & $0.0(0)$ & 1.7 & 0.08 \\
\hline Sclerosis & $0.0(0)$ & $1.9(3)$ & -1.4 & 0.15 \\
\hline Spasms & $2.8(3)$ & $1.2(2)$ & 0.9 & 0.37 \\
\hline Wasting & $1.8(2)$ & $0.6(1)$ & 0.9 & 0.35 \\
\hline Weight loss & $5.5(6)$ & $1.2(2)$ & 2.0 & 0.04 \\
\hline $\mathrm{ADD}$ & $5.5(6)$ & $3.7(6)$ & 0.7 & 0.49 \\
\hline AUD & $2.8(3)$ & $4.4(7)$ & -0.7 & 0.50 \\
\hline ALZ & $1.8(2)$ & $0.6(1)$ & 0.9 & 0.35 \\
\hline Anxiety & $39.5(43)$ & $26.7(43)$ & 2.2 & 0.03 \\
\hline Autism & $1.8(2)$ & $1.2(2)$ & 0.4 & 0.70 \\
\hline Depression & $20.2(22)$ & $16.2(26)$ & 0.9 & 0.39 \\
\hline Insomnia & $15.6(17)$ & $11.8(19)$ & 0.9 & 0.37 \\
\hline PTSD & $6.4(7)$ & $3.1(5)$ & 1.3 & 0.20 \\
\hline Recreation & $34.9(38)$ & $76.4(123)$ & -6.8 & $<0.001$ \\
\hline
\end{tabular}

Note: $G I=$ gastrointestinal problems; $A D D=$ attention deficit disorder; $A U D=$ alcohol use disorder; $A L Z=$ Alzheimer's disease; $P T S D=$ post-traumatic stress disorder; $Z$ derived from proportions test; statistically significant differences highlighted in bold (Bonferroni correct $p<.0015$ ). 


\section{RESULTS}

All analyses were conducted in Stata 16.1 (StataCorp, 2021). For items that were frequency counts (i.e., number of conditions endorsed, number of side effects), $t$-tests compared mean differences across CBD and cannabis. When considering single item frequencies (e.g., individual diagnoses, side effects), $z$ proportions were used to compare the percentage of the sample who used CBD or cannabis for that purpose. On average, CBD formats were more varied than for cannabis. Sublingual, topical, and liquid CBD formulations were more commonly endorsed than for cannabis (Table 2), whereas smoking was greater for cannabis than for CBD. Overall, more side effects were reported for cannabis than CBD. Cannabis use was associated with more reports of red eye, dry mouth, hunger/overeating, and increased pulse/heart rate than for CBD. Euphoria, generally a desired effect for cannabis, was substantially higher than for CBD. The average amount spent per week on CBD and cannabis varied greatly but did not differ between the two (Table 2). No statistically significant age group differences emerged for use patterns or side effects (Bonferroni $p<0.001$ ).

While the recreational use motive was more commonly endorsed for cannabis, use for beauty purposes was more common for CBD (Table 3). General health and well-being motives and endorsement of the specific use motives for medical/psychiatric conditions were commensurate for CBD and cannabis. Despite predictions, no age group differences emerged for motives to use CBD or cannabis (Bonferroni $p<$ 0.0008).

Pairwise correlations examined associations between use motives, daily cannabis use, and frequency of cannabis use. Daily use of cannabis correlated with use of cannabis for general medical reasons, $r=0.60, p=0.002$. Cannabis frequency was correlated with cannabis use for reasons of general health, $r=0.45, p<0.001$ and recreation, $r=0.38, p<0.001$. CBD use motives were not statistically related to either DFAQ-CU use metric.

\section{DISCUSSION}

This investigation examined differences between use patterns, use characteristics, and specific motives for $\mathrm{CBD}$ and cannabis use in a sample of adults over 21 living in recreational legal states in the U.S. As might be expected, the greatest differences between self-reported CBD and cannabis use were for effect profiles, given pharmacological differences between $\mathrm{CBD}$ and cannabis (Grotenhermen, 2003). Differences emerged for use formats with smoking for cannabis and sublingual, topical, and liquid formulations for CBD. Similar to the findings by Corroon and Phillips (2018), who found that the top conditions for which people used CBD were pain, anxiety and depression, and insomnia, the most frequently reported conditions for which participants used CBD were anxiety, depression, chronic pain management, and insomnia. Significantly, there was an overlap between the conditions for which cannabis and CBD were used, possibly due to the presence of $\mathrm{CBD}$ in cannabis. However, this requires further study, including items specific to dosing and $\mathrm{CBD} / \mathrm{THC}$ content. Overall, this study suggests that both cannabis and CBD are used to treat and manage medical and psychiatric symptoms as well as to promote general health and wellness.

We also examined possible age-related differences in motives to use CBD. Contrary to Haug et al. (2017), age group differences did not emerge here; sampling difference (ages 21-55+ vs. 18-74) may have led to differential findings. Future research is needed to further explore agerelated differences in a larger, more age-diverse samples. All cannabis use motives, except beauty/skincare, correlated with daily or frequency of cannabis use. Demonstrating discriminant validity, CBD use motives were not statistically related to either use metric.

The present study contributes to the emergent literature on CBD users in the U.S. The findings of the study provide insights into CBD use patterns and motives among a younger sample than previously described. Furthermore, this is the first study that examined health condition motives, including psychiatric disorders, for CBD and cannabis use separately and assessed nonmedical motives to use CBD. Some of the limitations of this study include the relative homogeneity of the sample, small sample size, and the use of self-report. Future studies with large, diverse samples of CBD and cannabis users should more fully evaluate the factor structure and construct validity of the checklist employed. 
The veracity of self-report for cannabis in MTurk samples has been questioned (Kim \& Hodgins, 2017); as such, caution should be used when interpreting these results. To extend the findings from the current study, participants under the age of 25 and participants over the age of 55 should be actively recruited to compare their motives for use. In order to get a more representative sample of cannabis and CBD users, participants from the states that have not yet legalized medical and recreational cannabis should be recruited. On balance, these results provide preliminary support for further examination of similarities and differences among users of CBD and cannabis for a variety of reasons, beyond simply those for self-medication.

\section{REFERENCES}

Bonn-Miller, M. O., Boden, M. T., Bucossi, M. M., \& Babson, K. A. (2014). Self-reported cannabis use characteristics, patterns and helpfulness among medical cannabis users. American Journal of Drug and Alcohol Abuse, 40(1), 23-30.

Bonn-Miller, M. O., Loflin, M. J. E., Thomas, B. F., Marcu, J. P., Hyke, T., \& Vandrey, R. (2017). Labeling accuracy of cannabidiol extracts sold online. JAMA, 318(17), 1708-1709.

Boulder, C. (2019, May 9). U.S. CBD marked anticipated to reach $\$ 20$ billion in sales by 2024 . Retrieved from www.bsanalytics.com

Corroon, J., \& Kight, R. (2018). Regulatory status of cannabidiol in the US: a perspective. Cannabis and Cannabinoid Research, 3(1), 190-194.

Corroon, J., \& Phillips, J. A. (2018). A cross-sectional study of cannabidiol users. Cannabis and Cannabinoid Research, 3(1), 152-161.

Cuttler, C., \& Spradlin, A. (2017). Measuring cannabis consumption: Psychometric properties of the Daily Sessions, Frequency, Age of Onset, and Quantity of Cannabis Use Inventory (DFAQCU). PLOS ONE, 12(5), e0178194.

ElSohly, M. A. and W. Gul (2014). Handbook of cannabis (Chapter 2). Oxford, UK: Oxford University Press: P.20. Retrieved from http://www.oxfordscholarship.com/view/10.1093/ acprof:oso/9780199662685.001.0001/acprof9780199662685

Fedorova, E. V., Wong, C. F., Ataiants, J., Iverson, E., Conn, B. M., \& Lankenau, S. E. (2021). Cannabidiol (CBD) and other drug use among young adults who use cannabis in Los
Angeles. Drug and Alcohol Dependence, 221, 108648.

Greenwich Biosciences, Inc. (2018). Epidiolex: highlights of prescribing information. Carlsbad, CA: Author.

Grotenhermen, F. (2003). Pharmacokinetics and pharmacodynamics of cannabinoids. Clinical Pharmacokinetics, 42(4), 327-360.

Haug, N. A., Padula, C. B., Sottile, J. E., Vandrey, R., Heinz, A. J., \& Bonn-Miller, M. O. (2017). Cannabis use patterns and motives: a comparison of younger, middle-aged, and older medical cannabis dispensary patients. Addictive Behaviors, 72, 14-20.

Kessler, R. C., Amminger, G. P., Aguilar-Gaxiola, S., Alonso, J., Lee, S., \& Ustun, T. B. (2007). Age of onset of mental disorders: a review of recent literature. Current Opinion in Psychiatry, 20(4), 359-364.

Kim, H.S. \& Hodgins, D.C. (2017). Reliability and validity of data obtained from alcohol, cannabis, and gambling populations on Amazon's Mechanical Turk. Psychology of Addictive Behaviors, 31(1), 85-94.

Larsen, C., \& Shahinas, J. (2020). Dosage, efficacy and safety of cannabidiol administration in adults: A systematic review of human trials. Journal of Clinical Medicine Research, 12(3), 129-141.

Leas, E. C., Nobles, A. L., Caputi, T. L., Dredze, M., Smith, D. M., \& Ayers, J. W. (2019). Trends in internet searches for cannabidiol (CBD) in the US. JAMA Network Open, 2(10), e1913853e1913853.

Lijster, J. M. de, Dierckx, B., Utens, E. M. W. J., Verhulst, F. C., Zieldorff, C., Dieleman, G. C., \& Legerstee, J. S. (2017). The age of onset of anxiety disorders. Canadian Journal of Psychiatry. Revue Canadienne De Psychiatrie, 62(4), 237246.

Miller, R. J., \& Miller, R. E. (2017). Is cannabis an effective treatment for joint pain? Clinical and Experimental Rheumatology, 35 Suppl 1075), 59-67.

National Academies of Sciences. (2017). The health effects of cannabis and cannabinoids: the current state of evidence and recommendations for research.

Russo, E. B. (2007). History of cannabis and its preparations in saga, science, and sobriquet. Chemistry \& Biodiversity, 4(8), 1614-1648. 
Sexton, M., Cuttler, C., Finnell, J. S., \& Mischley, L. K. (2016). A cross-sectional survey of medical cannabis users: patterns of use and perceived efficacy. Cannabis and Cannabinoid Research, 1(1), 131-138.

Shannon, S., Lewis, N., Lee, H., \& Hughes, S. (2019). Cannabidiol in anxiety and sleep: a large case series. The Permanente Journal, 23.

StataCorp (2019). Stata Statistical Software: Release 16. College Station, TX: StataCorp LLC.

U.S. Drug Enforcement Administration (n.d.). Drug Scheduling. Retrieved June $17^{\mathrm{h}}, 2020$, from https://www.dea.gov/drug-scheduling

Vedelago, L., Metrik, J., \& Amlung, M. (2020). Differentiating medicinal and recreational cannabis users via cannabis use motives. Cannabis, 3, 52-63.

Zenone, M. A., Snyder, J., \& Crooks, V. A. (2021). What are the informational pathways that shape people's use of cannabidiol for medical purposes? Journal of Cannabis Research, 3(1), 13.

Funding and Acknowledgements: Reed College Initiative Grant (A. Bilaonova).

Copyright: (C) 2021 Authors et al. This is an open access article distributed under the terms of the Creative Commons Attribution License, which permits unrestricted use, distribution, and reproduction, provided the original author and source are credited, the original sources is not modified, and the source is not used for commercial purposes.

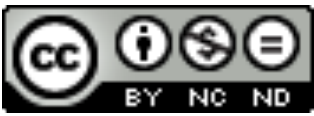

